\title{
Investigation of the Role of Mental Workload, Fatigue, and Sleep Quality in the Development of Musculoskeletal Disorders
}

\author{
Mohammad Babamiri ${ }^{1}$, Rashid Heidarimoghadam ${ }^{1}$, Hamid Saidnia', Younes \\ Mohammadi $^{3}$, Jamshid Joudaki ${ }^{2, *}$ \\ ${ }^{1}$ Health Sciences Research Center, Department of Ergonomics, School of Public Health, Hamadan University of Medical \\ Sciences, Hamadan, Iran \\ ${ }^{2}$ MA in Ergonomics, Department of Ergonomics, School of Public Health, Hamadan University of Medical Sciences, \\ Hamadan, Iran \\ ${ }^{3}$ Social Determinants of Health Research Center, Department of Epidemiology, School of Public Health, Hamadan University \\ of Medical Sciences, Hamadan, Iran
}

* Corresponding Author: Jamshid Joudaki, Department of Ergonomics, School of Public Health, Hamadan University of Medical Sciences, Hamadan, Iran. Email: jamshid.judaki@yahoo.com

Received: 02/09/2018 Accepted: 19/02/2019

How to Cite this Article: Babamiri M, Heidarimoghadam $R$, Saidnia H, Mohammadi $Y$, Joudaki J. Investigation of the Role of Mental Workload, Fatigue, and Sleep Quality in the Development of Musculoskeletal Disorders. J Occup Hyg Eng. 2019; 5(4): 1-7. DOI: 10.29252/ johe.5.4.1

\section{Abstract}

Background and Objective: Considering the importance and high prevalence of musculoskeletal disorders, this study aimed to investigate the role of mental workload, fatigue, and sleep quality in the development of musculoskeletal disorders.

Materials and Methods: This study included 243 employees working at Borujerd health center. The participants were selected based on simple random sampling. The data were collected using the Cornell Musculoskeletal Disorders Questionnaire, Sophie Fatigue Questionnaire, and Pittsburgh Sleep Quality Questionnaire. Moreover, the Subjective Workload Assessment Technique was utilized to evaluate the mental workload. The data were analyzed using SPSS software (Version 18) through the Pearson correlation coefficient, the Chi-square test, and regression analysis.

Results: According to the results of this study, back pain was ranked as the most prevalent disorder. The results of the correlation analysis showed a significant association between mental workload and fatigue with musculoskeletal disorders $(P \leq 0.05)$. Moreover, a significant relationship was observed between sleep quality and musculoskeletal disorders $(P \leq 0.05)$. Conclusion: It can be concluded that reduced workload and fatigue, as well as improved sleep quality, can decrease the risk of musculoskeletal disorders among the staff.

Keywords: Fatigue; Mental workload; Musculoskeletal Disorder; Sleep Quality 
do: : 10.29252/johe.5.4.1

بررسى نقش بار كارى ذهنى، خستغى و كيفيت خواب در ايجاد اختلالات اسكلتى - عضلانى محمد باباميرى'، رشيد حيدرى مقدم'، حميد سعيدنيا'، يونس محمدى '، جمشيد جودكى r.* ' مركز تحقيقات علوم بهداشتى، كروه اركونومى، دانشكده بهداشت، دانشكاه علوم يزشكى همدان، همدان، ايران

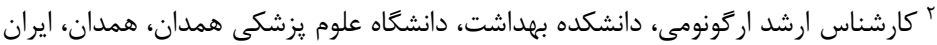

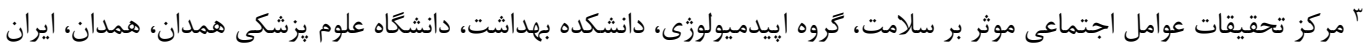
* نويسنده مسئول: جمشيد جودكى، دانشكده بهداشت، دانشخاه علوم يزشكى همدان، همدان، ايران. ايميل:yamshid.judaki@yahoo.com

جكيده

سابقه و هدف: با توجه به اهميت و شيوع بالاى اختلالات اسكلتى - عضلانى (Musculoskeletal Disorder)،

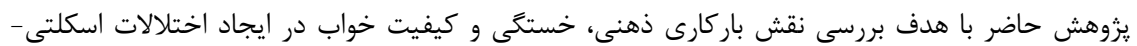
عضلانى انجام شد.

مواد و روشها: جامعه يزوهش حاضر كارمندان شاغل در مركز بهر بهداشت شهر بروجرد بودند كه براساس

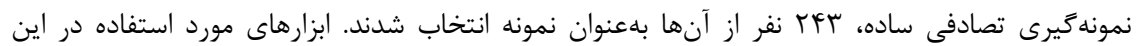

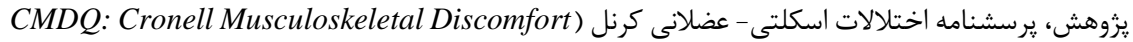
و (SOFI: Swedish Occupational Fatigue Inventory (Questionnaire يرسشنامه كيفيت خواب ييتسبورى (PSQI: Pittsburgh Sleep Quality Questionnaire) بودند.

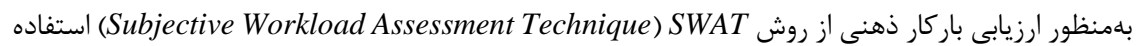

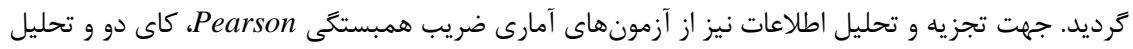

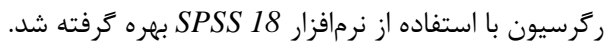

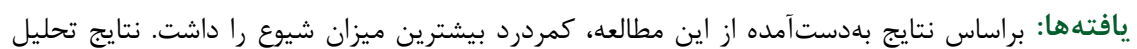

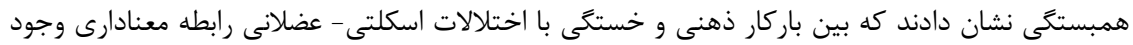

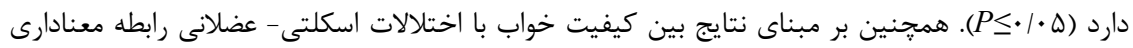

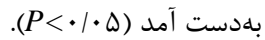

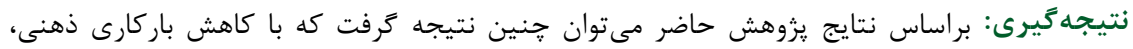

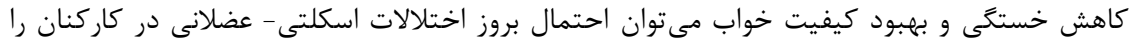
كاهش داد.

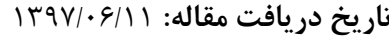

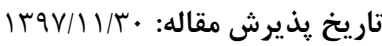
تمامى حقوق نشر براى دانشعاه علوم يزشكى همدان محفوظ است.

وازًَان كليدى: اختلالات اسكلتى - عضلانى؛ باركارى ذهنى؛ خستكى؛ كيفيت خواب

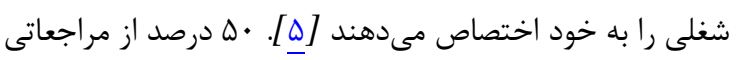
كه به يزشكان مىشود، به دليل بيمارىهاى إسى اسكلتى - عضلانى

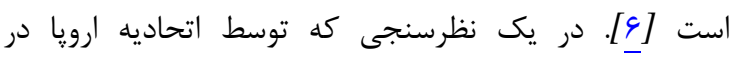

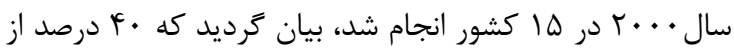

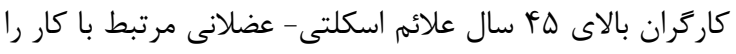

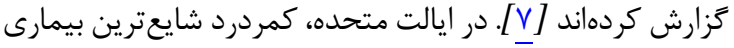

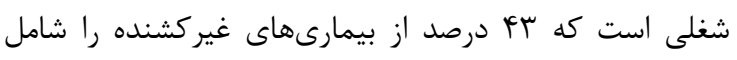
مىشود [^]ـ. يكى از عوامل تأثير حذار بر رفتار و عملكرد يرستاران

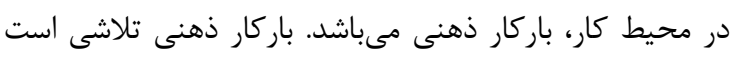

در ميان گروههاى مختلف شغلى در بخش سلامت به دليل

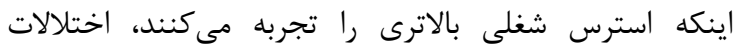

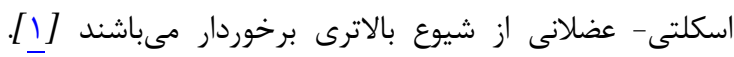

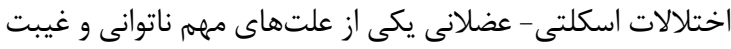

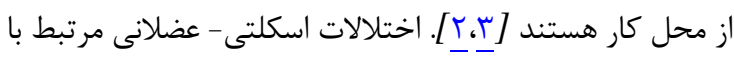

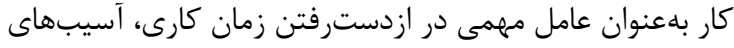

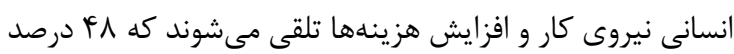

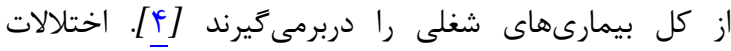

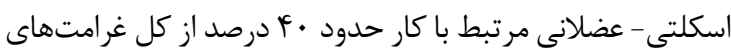


با توجه به آنجه كه كَفته شد باركارى، خستكى و كيفيت

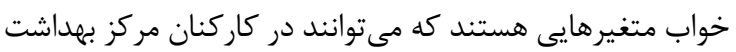

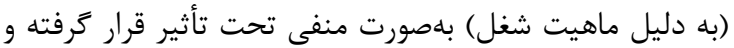

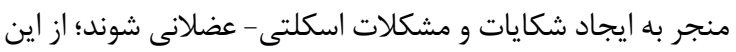
رو در جهت مشخصشدن هرجه بهتر اين رابطه، يزوهش حاضر

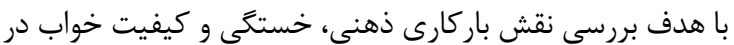

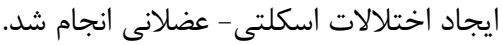

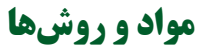

يزوهش مقطعى حاضر در سال 99 ا در مورد كاركنان مركز

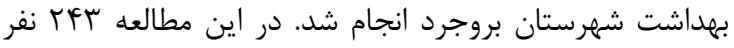

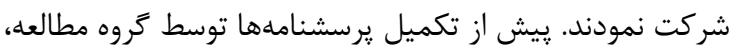
اهداف اصلى يزوهش براى شركت كنند كان توضيح داده شد و فرم برم

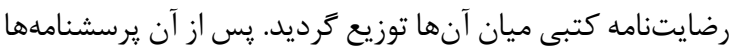

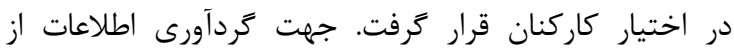
يرسشنامههاى زير استفاده گرديد:

\section{يرسشنامه ناراحتى اختلالات اسكلتى- عضلانى كرنل}

(CMDQ)

اين يرسشنامه در سال 1999 توسط Hedge و همكاران

تدوين شد و براى مطالعات مقطعى استفاده گرديد. در اين

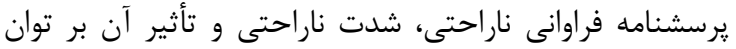

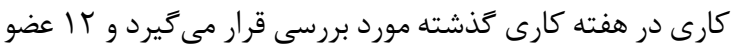

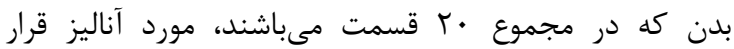
مى گيرند. در مطالعهاى كه توسط عفيفهزاده و همكاران بهمنظور

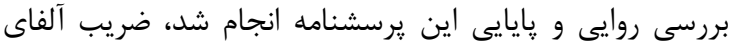

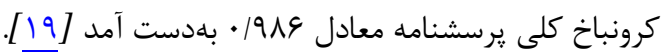

\section{يرسشنامه بررسى خستتسى شغلى (SOFI)}

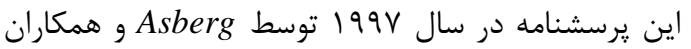

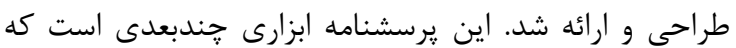

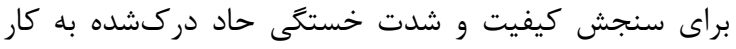

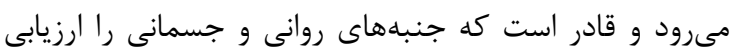

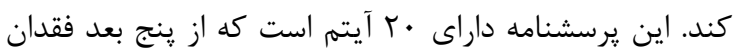

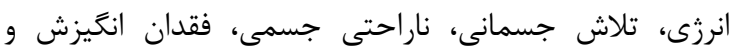

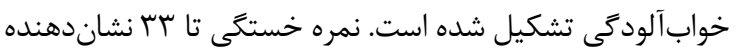
خستكى كم، نمره צY-4 9V به بالا نشاندهنده خستخى بالا مىباشد. بايد خاطرنشان

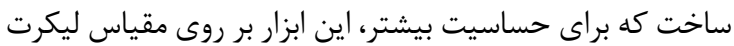

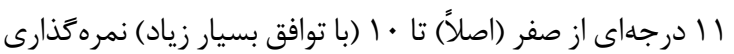
مىشود. در مطالعهاى كه توسط جواديور و همكاران در سال

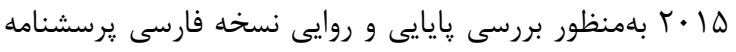
SOFI

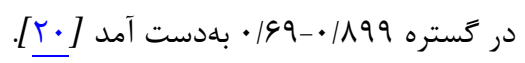

كه ذهن در حين انجام وظيفه انجام مىدهد و بلصورت"سطحى

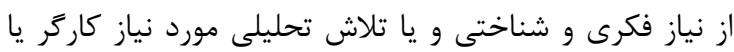

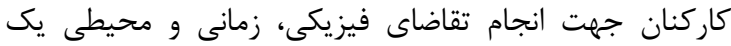
وظيفه مشخص "تعريف شده است. طاهرى و حبيبى در دران

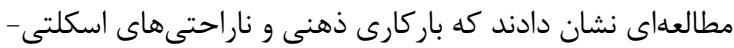

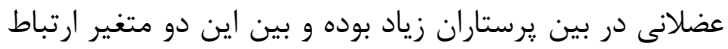

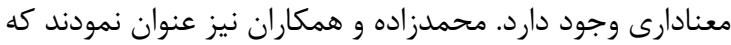

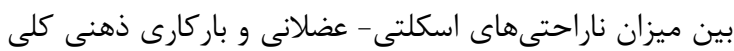

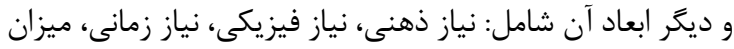

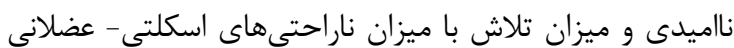

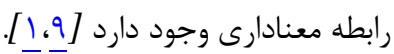

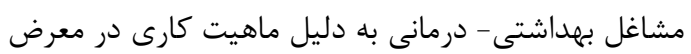
ريسك بالايى براى خستخى (Fatigue) و بلويزه اختلالات

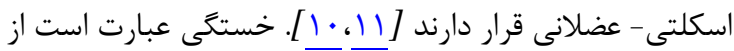

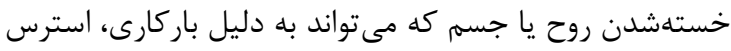

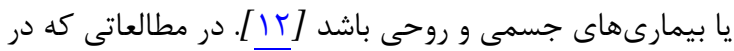

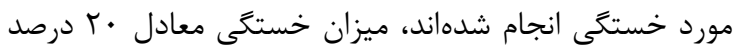

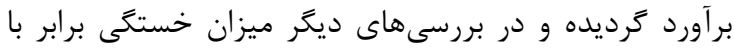

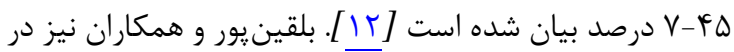

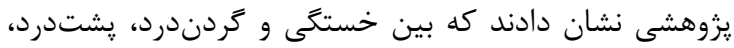

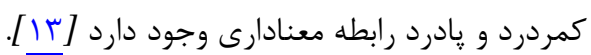

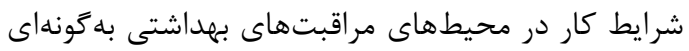

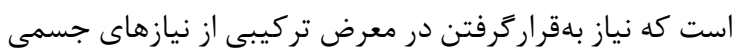

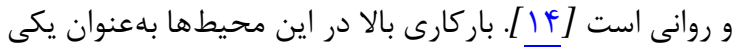

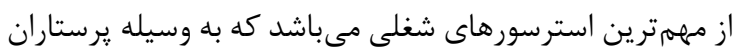

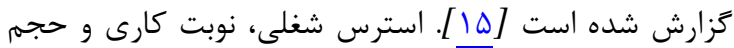

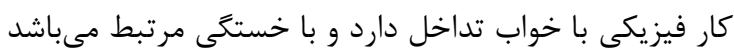

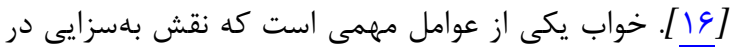

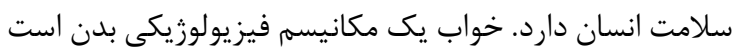

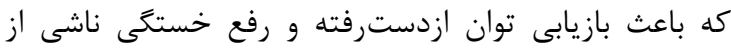
فعاليت مىشود. هر گونه اختلال در خواب انسان مى تواند مندر

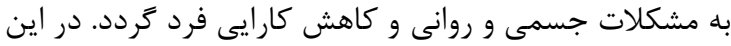
راستا در مطالعهاى كه توسط Tekeoglu و همكاران انجام شد، مشخص گرديد كه بين نمره درد ارزيابىشده با كيفيت ذهنى خواب (Subjective Sleep Quality)، تأخير خواب، مدت كرديد

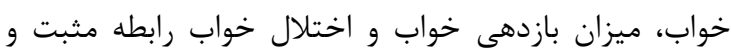

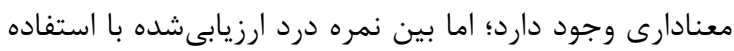

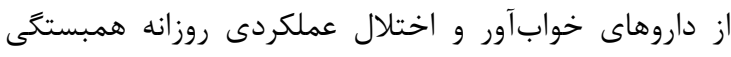

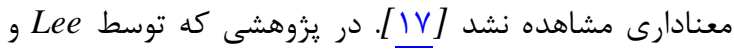

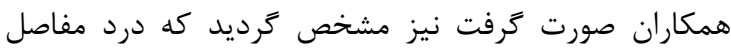

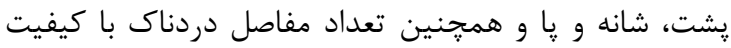

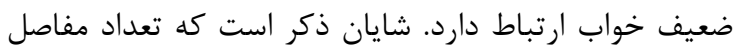

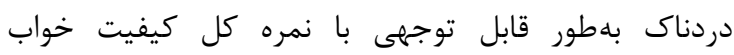
همبستخى مثبت داشت [11 دائ. 
يرسشنامه است (بهطور مثال نمره ا به عدد ال 111 در جدول

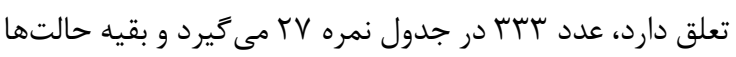

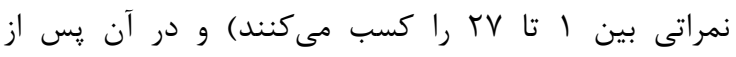

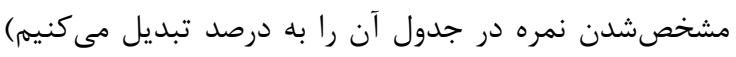

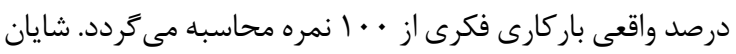

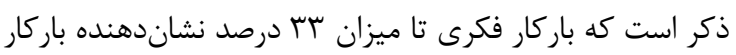

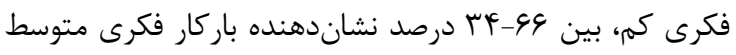

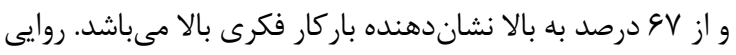

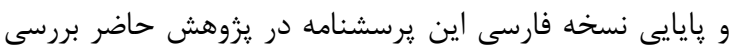

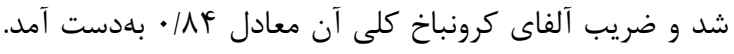

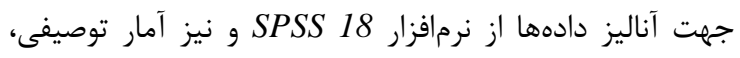

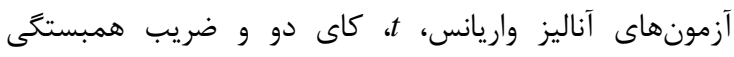
Pearson در سطح معنادارى ه • • • استفاده گرديد.

\section{كافته.}

مطالعه حاضر در ارتباط با سعب نفر از كاركنان مركز بهداشت صورت كرفت. ميانكين سنى شركت كنند r ا نفر از شركت كنند درصد) مرد بودند. از نظر توزيع جمعيت بر حسب اند مين ميزان

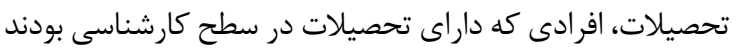

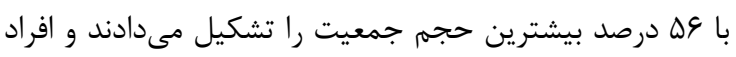

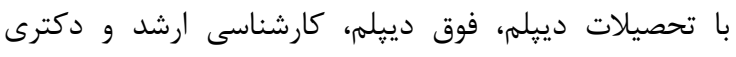

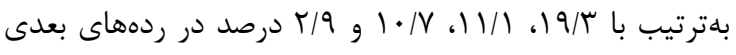

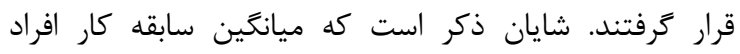

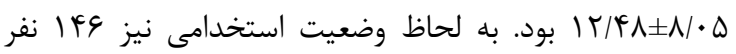

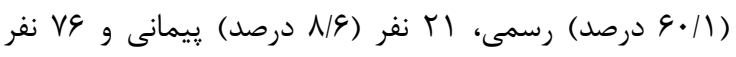

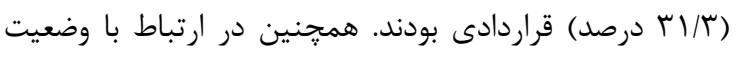

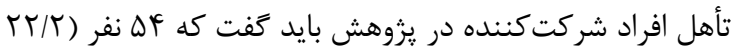

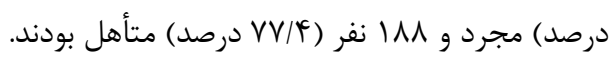

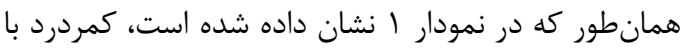

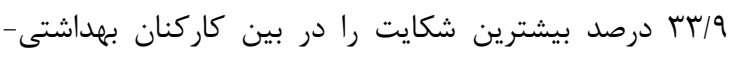

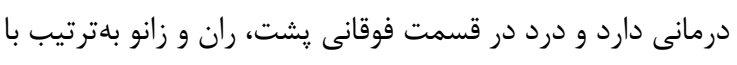

برسشنامه كيفيت خواب بيتسبورت (PSQI)

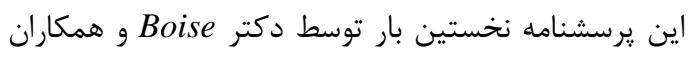

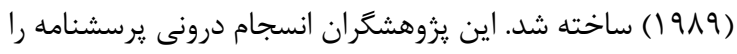

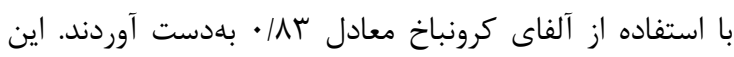
مقياس شامل هفت مؤلفه مىباشد كه عبارت هستند از: كيفيت ذهنى خواب، مدت زمان خواب، تأخير در خواب، ميزان بازدهي مئى

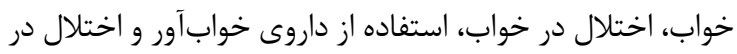

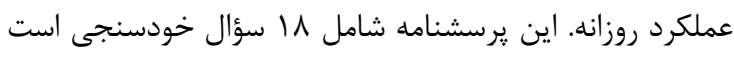
كه تركيبى از هفت مؤلفه هستند و دامنه هريك از آنها در يك رئن

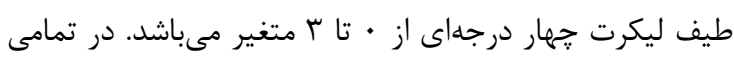

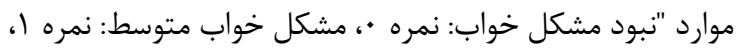

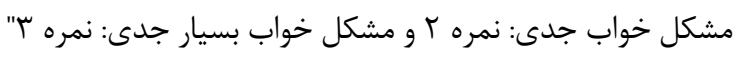
را نشان مى دهد. تركيب نمرات هفت مؤلفه با يكديكر، يك نمره ندره

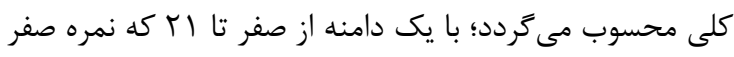

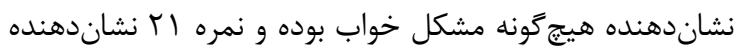
جندين مشكل در تمامى محدودهها مىباشد. روايى و ويايايى اين

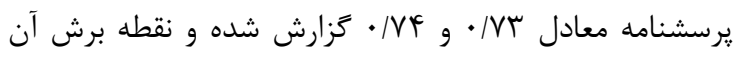

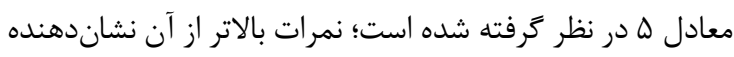
نامطلوببودن كيفيت خواب مىباشد [ [r]].

\section{يرسشنامه باركارى ذهنى (SWAT)}

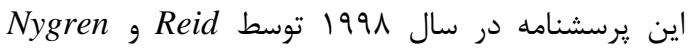

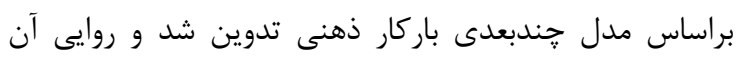

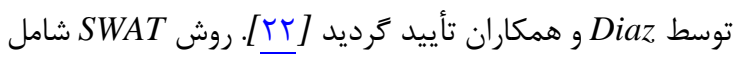

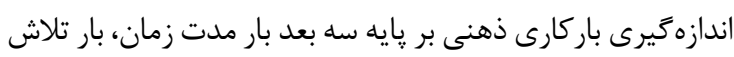

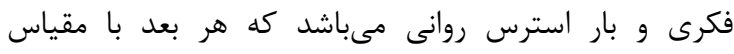

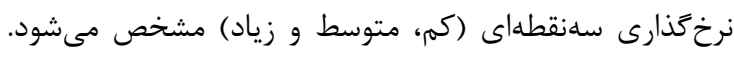

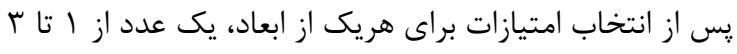

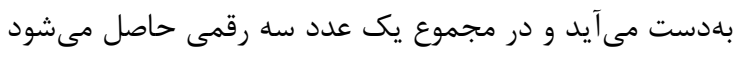

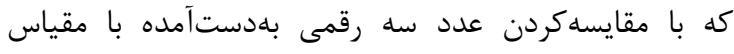

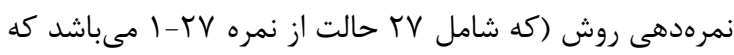
هر حالت مشخص كننده يكى از اعداد سه رقمى بهدست آمده آمداز

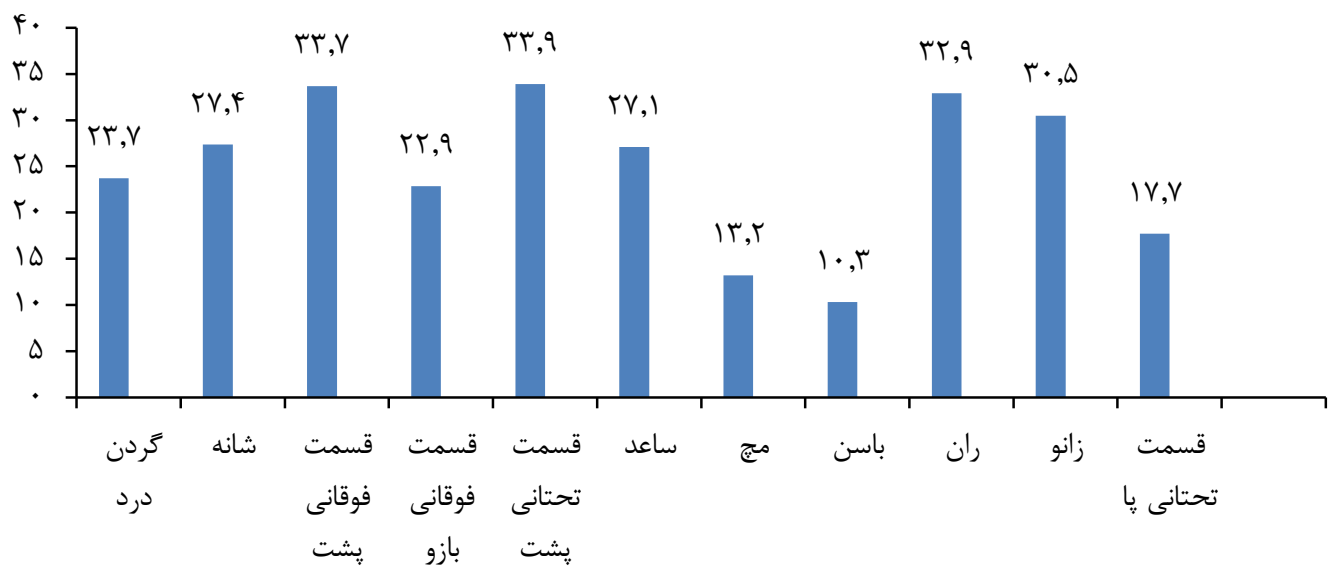

نمودار ا: توزيع فراوانى ناراحتى هاى اسكلتى عضلانى در بين كاركنان بلن بلن 


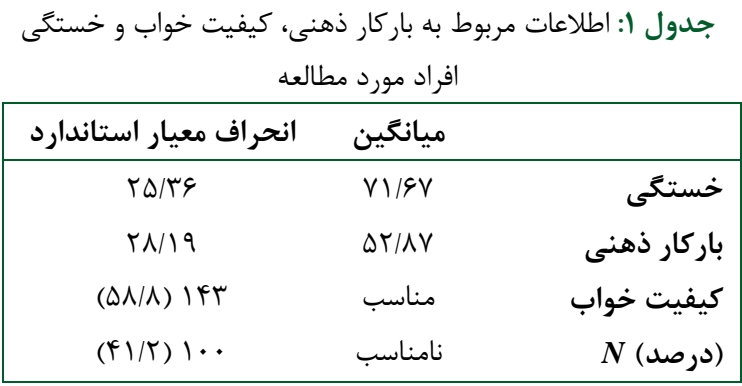

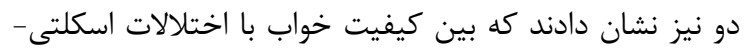
عضلانى رابطه معنادارى وجود دارد (ه •/• > (P).

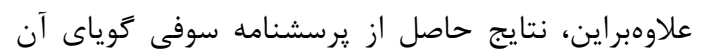

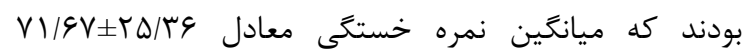

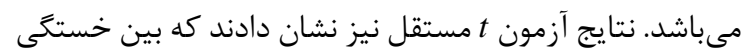

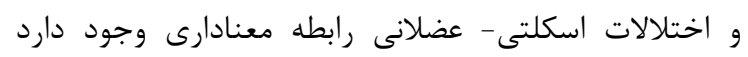

$$
\text { . }(P<\cdot / \cdot \Delta)
$$

شكا شكايت را به خود اختصاص دادهاند.

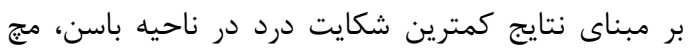

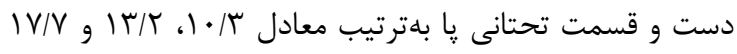

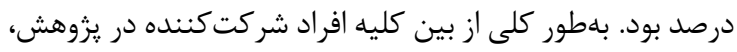

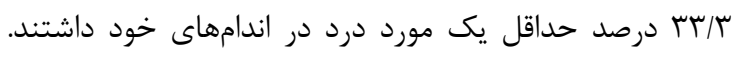

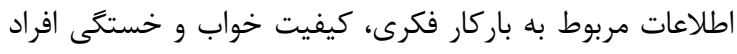

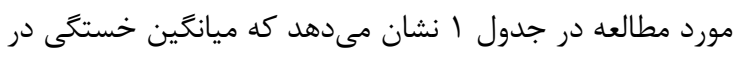

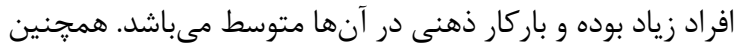

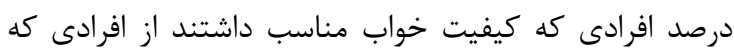
داراى كيفيت خواب نامناسب بودند، بيشتر است

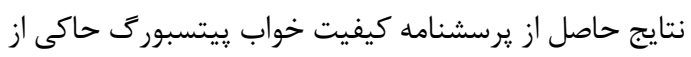

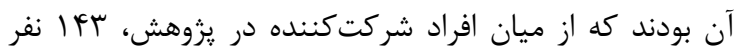

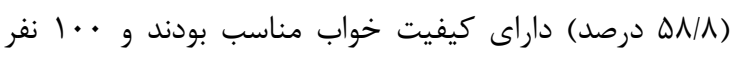

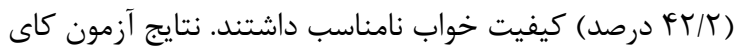

جدول ז: رابطه بين باركار ذهنى، خستكى و كيفيت خواب با اختلالات اسكلتى - عضلانى

\begin{tabular}{|c|c|c|c|}
\hline كيفيت خواب & باركار ذهنى & خستخى & اختلالات اسكلتى - عضلانى \\
\hline ( & $\cdot / \cdot \mu^{*}$ & 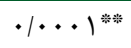 & درد كردن \\
\hline$\cdot 199$ & - IAts & $\cdot / \cdots)^{\text {漛 }}$ & درد شانه راست \\
\hline$\cdot / \cdot V$ & .1 .4 & $\cdot / \cdot r^{* * *}$ & درد شانه جٍ \\
\hline$\cdot / r v$ & $\cdot / 1 V 9$ & $\cdot|\cdots|^{\text {䒜 }}$ & درد قسمت فوقانى يشت \\
\hline$\cdot / \Delta T$ & $\cdot / r \wedge F$ &.$/ 1 r q$ & درد قسمت فوقانى بازوى راست \\
\hline$\cdot / 49$ & $\cdot / r \cdot r$ & $\cdot 1 \cdot 1^{*}$ & درد قسمت فوقانى بازوى جٍ \\
\hline$\cdot 11$ &.$/ 999$ & 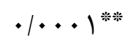 & درد قسمت تحتانى يشت \\
\hline .109 & $\cdot \mid \Delta \Delta F$ & $\cdot 1 \cdot 1$ & درد ساعد راست \\
\hline$\cdot / 4 q$ & - IGTY & .119 & درد ساعد ج" \\
\hline$\cdot / r \Delta \Lambda$ & .1011 & 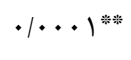 & درد مج دست راست \\
\hline$\cdot / 49$ & $\cdot / r \wedge r$ & $\cdot|\cdot \cdot|^{* * * *}$ & درد مج دست ج" \\
\hline •/Ve & $\cdot / r \Delta q$ & - /Arq & درد باسن \\
\hline$\cdot / 4 V$ & .1199 & $\cdot / \cdot r^{\text {***** }}$ & درد ران راست \\
\hline$\cdot 1 \cdot 1^{*}$ & $.194 \mathrm{r}$ & $\cdot / \cdot r^{*}$ & درد ران حٍ \\
\hline$\cdot / \cdot r^{*}$ & - I M & $\cdot / \cdot \varphi^{*}$ & درد زانوى راست \\
\hline .1 .9 & - IAST & $\cdot / \cdot \Delta^{* * *}$ & درد زانوى جֶٍ \\
\hline$\cdot / 14$ & $\cdot / F \Delta V$ & $\cdot / \cdot r^{*}$ & درد قسمت تحتانى پِى راست \\
\hline$\cdot 110$ & $\cdot \pi T V$ & $\cdot / \cdot \varphi^{*}$ & 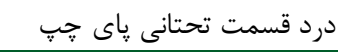 \\
\hline
\end{tabular}

جدول با: رابطه بين متغيرهاى كيفيت خواب، استرس و بار كار ذهنى با شدت كلى اختلالات اسكلتى - عضلانى

\begin{tabular}{|c|c|c|c|c|}
\hline بار كار ذهنى & خستخى & كيفيت خواب & & متغير \\
\hline$+\cdot 1 \cdot 9$ & $+\cdot / K F$ & $+\cdot / 10$ & ميزان رابطه & \\
\hline$P<\cdot / \cdot \Delta$ & $P<\cdot / \cdot \Delta$ & $P<\cdot / \cdot \Delta$ & سطح معنادارى & 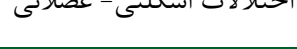 \\
\hline
\end{tabular}

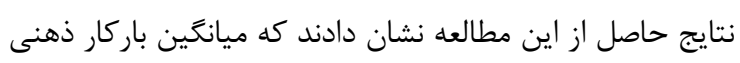

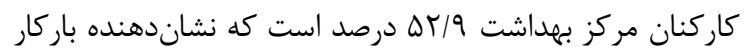

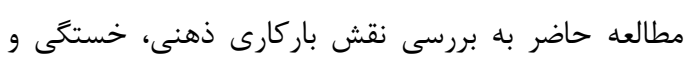

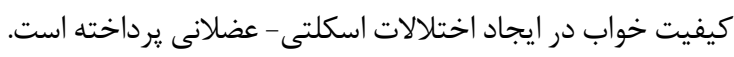


كه توسط عباسى و همكاران در مورد بررسى رابطه بين كيفيت

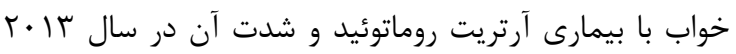

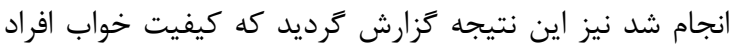

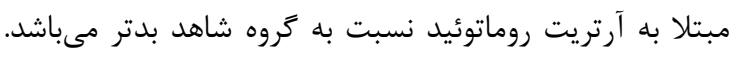

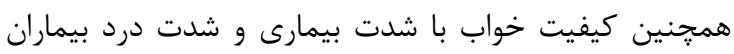

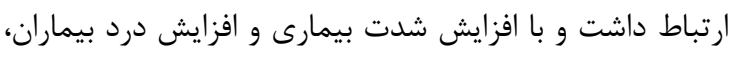

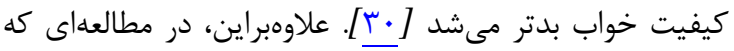

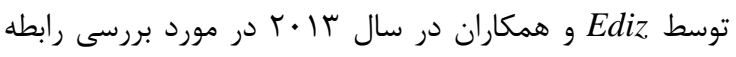

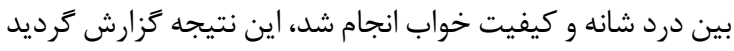

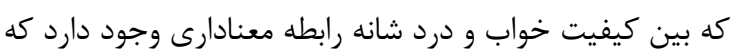

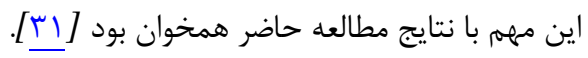

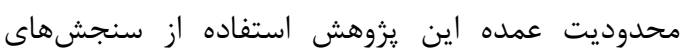

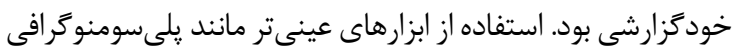

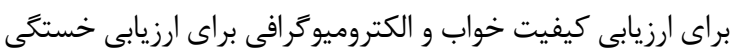

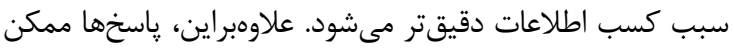
است به وسيله جوابهاى نادرست تحت تأثير قرار كيرند. براى

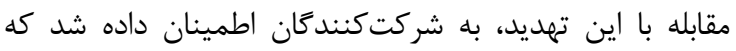

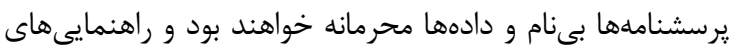

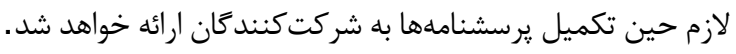

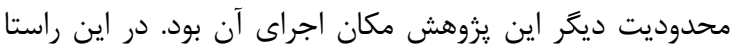

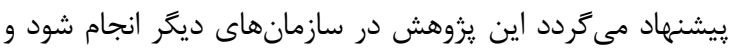
نتايج آن با يزوهش حاضر مقايسه كردد.

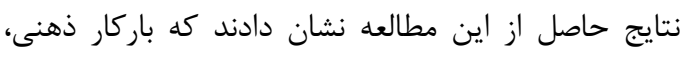
خستكى و كيفيت خواب نامناسب، ريسكفاكتورهاى اختلالات اسكلتى - عضلانى مى باشند و تعديل يا حذف ريسكفيت رئاكتورهاى

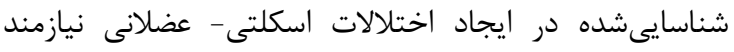

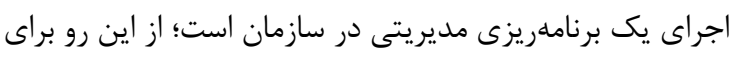

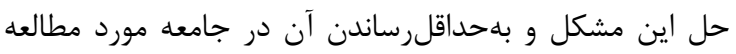

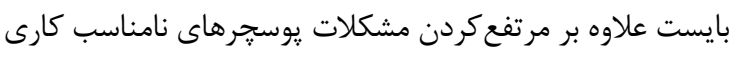

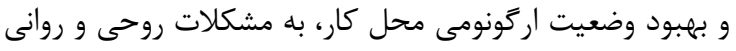

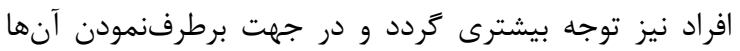

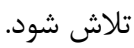

\section{نتيجه تيرى}

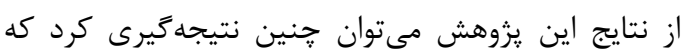
كاهش باركارى ذهنى، كاهش خستكى و بهبود كيفيت خواب ترني

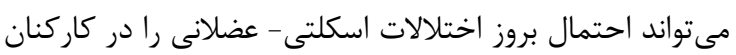

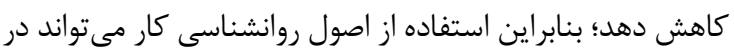

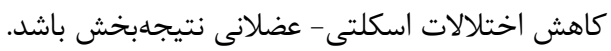

\section{تشكر و قدردانى}

بدينوسيله نويسند كان مراتب تقدير و تشكر خود را از تمامى شركت كنندگان در اين يزوهش و نيز افرادى كه آنها راد در انجام اين يزوهش يارى رساندند، اعلام مىدارند.
فكرى متوسط مىباشد. در اين مطالعه با توجه به آزمون كاى دو

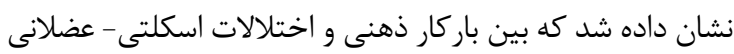

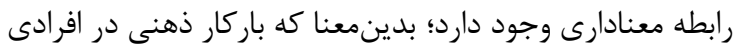

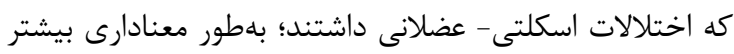

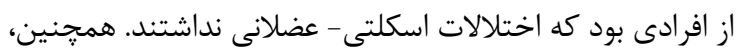

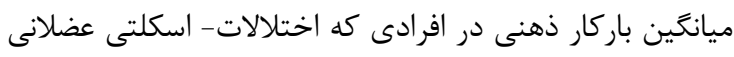

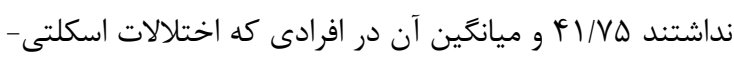

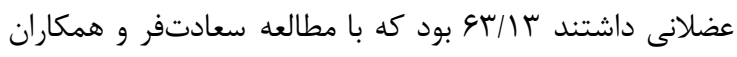
كه در مورد بررسى ارتباط باركارى با بيمارىهاى اسكلتى دانس

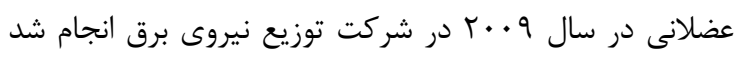

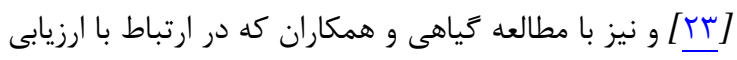

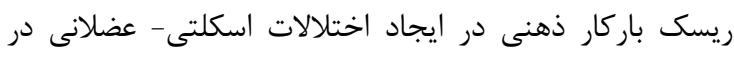

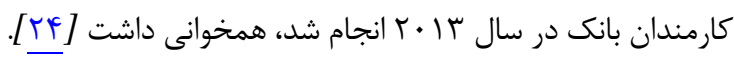

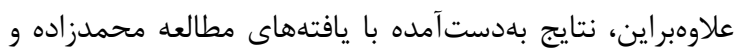

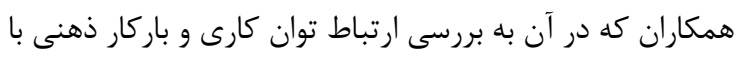

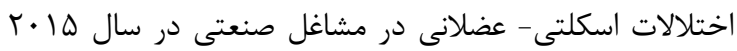

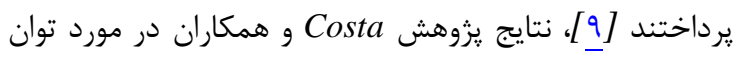

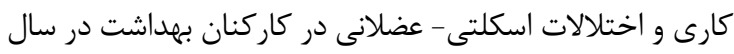

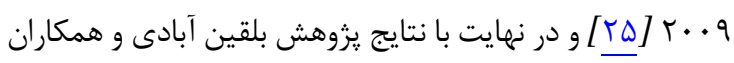

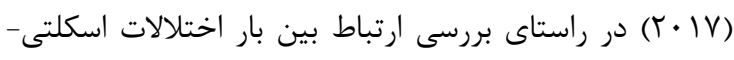

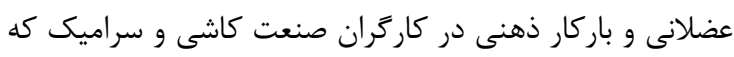

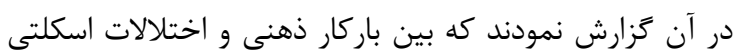

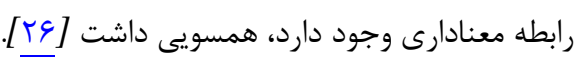

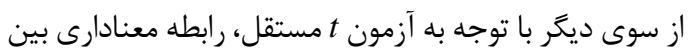

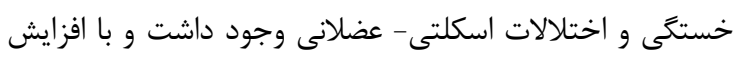

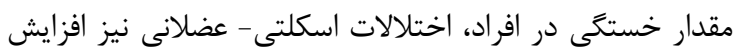

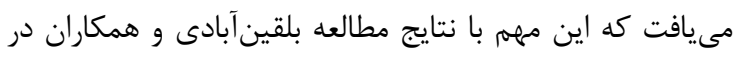

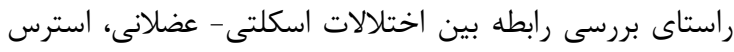
شغلى و خستكى در كاركنان يك صنعت مواد غذايى در سال

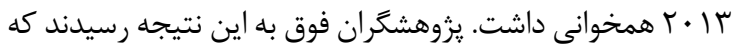

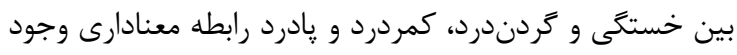

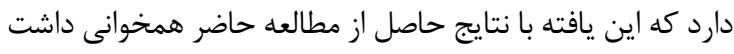

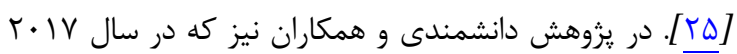

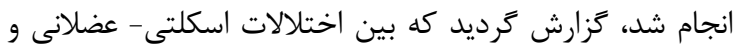

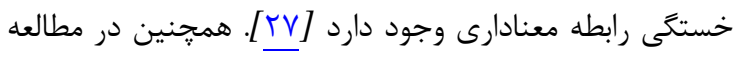
Shattuck

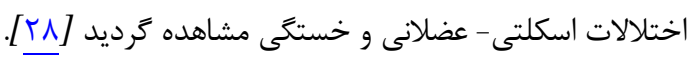

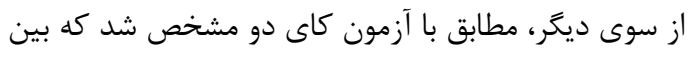

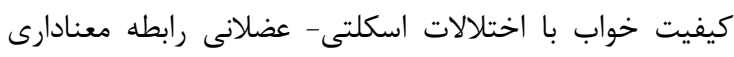

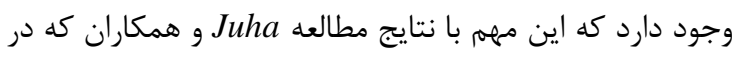

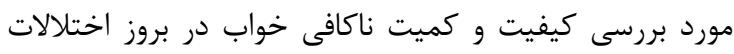

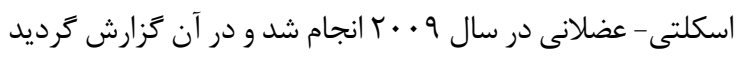
كه كردندرد و شانهدرد در افرادى كه كميت و كيفيت خواب آنساب

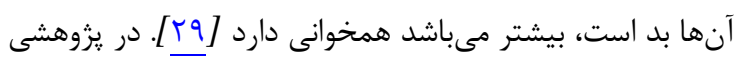




\section{REFERENCES}

1. Taheri M, Habibi A, Hasanzadeh A, Mahdavirad M. Relationship mental workload with musculoskeletal disorders among Alzahra hospital nurses by NASA-TLX index and CMD. J Health Syst Res. 2014;10(4):775-85. [Persian]

2. Waters TR. National efforts to identify research issues related to prevention of work-related musculoskeletal disorders. J Electromyogr Kinesiol. 2004;14(1):7-12. PMID: 14759745 DOI: 10.1016/j.jelekin.2003.09.004

3. Walsh I, Corral S, Franco R, Canetti E, Alem M, Coury $H$. Work ability of subjects with chronic musculoskeletal disorders. Rev Saude Publica. 2004;38(2):149-56. PMID: 15122368

4. Rahimi Fard H, Hashemi Nejad N, Choobine A, Heidari H, Tabatabaee S. Risk factors assessment cause musculoskeletal disorders in painting workshops of furniture industry. Qom Univ Med Sci J. 2011;4(2):35-45. [Persian]

5. Stansfeld SA, North F, White I, Marmot MG. Work characteristics and psychiatric disorder in civil servants in London. J Epidemiol Community Health. 1995;49(1):48-53. PMID: 7707005

6. Pincus T, Burton AK, Vogel S, Field AP. A systematic review of psychological factors as predictors of chronicity/disability in prospective cohorts of low back pain. Spine. 2002;27(5):E109-20. PMID: 11880847

7. Ilmarinen $J$. The ageing workforce--challenges for occupational health. Occup Med. 2006;56(6):362-4. PMID: 16931565 DOI: 10.1093/occmed/kql046

8. Cole D, Ibrahim S, Shannon HS, Scott F, Eyles J. Work correlates of back problems and activity restriction due to musculoskeletal disorders in the Canadian national population health survey (NPHS) 1994-5 data. Occup Environ Med. 2001;58(11):728-34. PMID: 11600729

9. Mohammadzadeh M, Habibi A, Hasanzadeh A. Relationship between work ability and mental workload with musculoskeletal disorders in industrial jobs. J Prev Med. 2015;2(4):29-38. [Persian]

10. Choobineh A, Rajaeefard A, Neghab M. Association between perceived demands and musculoskeletal disorders among hospital nurses of Shiraz University of Medical Sciences: a questionnaire survey. Int J Occup Saf Ergon. 2006;12(4):409. 16. PMID: 17156616 DOI: 10.1080/10803548.2006.11076699

11. Menzel NN. Psychosocial factors in musculoskeletal disorders. Crit Care Nurs Clin North Am. 2007;19(2):14553. PMID: 17512470 DOI: 10.1016/j.ccell.2007.02.006

12. Moradifar $R$, Hoveidi $H$, Givehchi S. Fatigue management and related issues of working environment. J Soc Issues Hum. 2015;3(8):15-8. [Persian]

13. Bolghanabadi S, Pour $M$. The relationship between musculoskeletal disorders, stress and fatigue in the food industry employees. Iran J Ergon. 2014;2(1):54-63. [Persian]

14. Nimbarte AD, Al Hassan MJ, Guffey SE, Myers WR. Influence of psychosocial stress and personality type on the biomechanical loading of neck and shoulder muscles. Int J Indust Ergon. 2012;42(5):397-405. DOI: 10.1016/j. ergon.2012.05.001

15. Kiekkas P, Sakellaropoulos GC, Brokalaki H, Manolis E, Samios A, Skartsani $C$, et al. Association between nursing workload and mortality of intensive care unit patients. J Nurs Scholarsh. 2008;40(4):385-90. PMID: 19094155 DOI: 10.1111/j.1547-5069.2008.00254.x

16. Akerstedt T, Fredlund P, Gillberg M, Jansson B. Work load and work hours in relation to disturbed sleep and fatigue in a large representative sample. J Psychosom Res. 2002; 53(1):585-8. PMID: 12127175

17. Tekeoglu I, Ediz L, Hiz O, Toprak M, Yazmalar L, Karaaslan
$G$. The relationship between shoulder impingement syndrome and sleep quality. Eur Rev Med Pharmacol Sci. 2013;17(3):4-7. PMID: 23426540

18. Li JJ, Appleton SL, Gill TK, Vakulin A, Wittert GA, Antic NA, et al. Musculoskeletal joint pain in men is not associated with obstructive sleep apnea or daytime sleepiness but is associated with poor sleep quality. Arthrit Care Res. 2017;69(5):742-7. DOI: 10.1002/acr.22994

19. Afifehzadeh-Kashani H, Choobineh A, Bakand S, Gohari MR, Abbastabar H, Moshtaghi $P$. Validity and reliability of farsi version of Cornell Musculoskeletal Discomfort Questionnaire (CMDQ). Iran Occup Health J. 2009;7(4):6975. [Persian]

20. Javadpor F, Keshavarzi S, Chobineh A, Aghabigi M. Reliability and validity of the Persian version of Swedish occupational fatigue questionnaire (SOFI) in Iran's labor. Iran J Ergon. 2015;3(1):50-8. [Persian]

21. Lima P, Medeiros A, Araujo J. Sleep-wake pattern of medical students: early versus late class starting time. Braz J Med Biol Res. 2002;35(11):1373-7. PMID: 12426638

22. Rubio S, Díaz E, Martín J, Puente JM. Evaluation of subjective mental workload: a comparison of SWAT, NASATLX, and workload profile methods. Appl Psychol. 2004;53(1):61-86. DOI: 10.1111/j.1464-0597.2004.00161.x

23. Saadatfar A, Ranjbarian M, Saremi M, Hashemian AH, Yazdian A. Risk assessment of musculoskeletal disorders in linemen of electric power distribution company of Kermanshah province using REBA method in 2015. J Rafsanjan Univ Med Sci. 2016;15(7):593-606. [Persian]

24. Giahi $O$, Darvishi $E$, Akbarzadeh $M$, Shahsavari $S$. Assessment of the relationship of the risk of subjective work load to musculoskeletal disorders in bank staff in Kurdistan Province. Sci J Kurdistan Univ Med Sci. 2013;19(4):36-45. [Persian]

25. Monteiro MS, Alexandre NM, Ilmarinen J, Rodrigues CM. Work Ability and musculoskeletal disorders among workers from a public health institution. Int J Occup Saf Ergon. 2009;15(3):319-24. PMID: 19744373 DOI: 10.1080/10803548.2009.11076813

26. Bolghanabadi S, Nayerabadi A, Taheri M. Relationship of musculoskeletal disorders with workload among the workers of a ceramic and tile factory in Neyshabur, Iran, in 2017. J Health Res Commun. 2017;3(3):25-33. [Persian]

27. Daneshmandi H, Choobineh AR, Ghaem H, Alhamd M, Fakherpour A. The effect of musculoskeletal problems on fatigue and productivity of office personnel: a cross-sectional study. J Prev Med Hyg. 2017;58(3):252-8. [Persian]

28. Lewis Shattuck $N$, Matsangas P, Moore J, Wegemann L. Prevalence of musculoskeletal symptoms, excessive daytime sleepiness, and fatigue in the crewmembers of a U.S. Navy Ship. Mil Med. 2016;181(7):655-62. PMID: 27391619 DOI: 10.7205/MILMED-D-15-00279

29. Auvinen JP, Tammelin TH, Taimela SP, Zitting PJ, Järvelin $M R$, Taanila AM, et al. Is insufficient quantity and quality of sleep a risk factor for neck, shoulder and low back pain? A longitudinal study among adolescents. Eur Spine J. 2009;19(4):641-9. PMID: 19936804 DOI: 10.1007/s00586009-1215-2

30. Abbasi M, Yazdi Z, Dizaniha M. Relationship between sleep quality and severity of rheumatoid arthritis. J Qazvin Univ Med Sci. 2013;17(4):32-8. [Persian]

31. Tekeoglu I, Ediz L, Hiz O, Toprak M, Yazmalar L, Karaaslan $G$. The relationship between shoulder impingement syndrome and sleep quality. Eur Rev Med Pharmacol Sci. 2013;17(3):370-4. PMID: 23426540 